\title{
EL ESTUDIANTADO DIGITAL COMO CENTRO DE LOS PROCESOS DE ENSEÑANZA. ANÁLISIS EN EDUCACIÓN SUPERIOR ANTE LA EMERGENCIA DEL ELEARNING
}

\section{DIGITAL STUDENTS AS THE CENTER OF TEACHING PROCESSES. AN ANALYSIS IN HIGHER EDUCATION BEFORE THE EMERGENCE OF E-LEARNING}

\author{
Pablo Rivera Vargas*, Cristina Alonso Cano**
}

\begin{abstract}
Resumen
La emergencia y dinámica del Elearning (o Formación Virtual), en los actuales escenarios de educación superior, evidencia una importante transformación acontecida en el marco de la sociedad de la información: la utilización de las Tics en los procesos de construcción del conocimiento. Este cambio ha ido de la mano, en primer lugar, de la mejora en la calidad y la oferta formativa existente, es decir, una oferta que ha buscado ser más inclusiva y representativa de las actuales demandas sociales. $Y$ en segundo lugar, se trata de una modalidad que ha intentado adaptarse a las actuales características del estudiantado digital y del digitalizado (Prensky, 2006), cuyo rol en los proceso de enseñanza, tiende a ser más activo y protagónico que en los modelos de formación tradicional. El presente trabajo, aborda esta segunda dimensión, analizando como el Elearning intenta ser un elemento de mediación entre las transformaciones actuales que se dan en la educación superior y la emergencia de un nuevo estudiantado virtual, cada vez más activo y autónomo.
\end{abstract}

Palabras clave: Elearning, sociología de la educación, modernidad, postmodernidad, estudiante virtual, sociedad de la información.

\begin{abstract}
The emergence and dynamics of E-learning (or Virtual Training) in the current scenarios of higher education evidences a major transformation that occurred in the context of the information society: the use of ICTs in the processes of knowledge construction. This change has gone hand in hand with the improvement in the quality and at the existing training offer, i.e. an offer that has sought to be more inclusive and representative of the current social demands. It is a modality that has tried to adapt to the current features of digital and digitized students (Prensky, 2006), whose role in the teaching process tends to be more active and more prominent than in traditional training models. The present study addresses this second dimension, analyzing how the E-learning aims to become an element of mediation between the current changes taking place in higher education and the emergence of new virtual students, who are increasingly more active and more independent.
\end{abstract}

Key words: E-learning, Sociology of Education, Modernity, Postmodernism, Virtual Student, Information Society

Artículo recibido: 28/06/2013 Aprobado: 31/07/2013

* Investigador. Universitat Oberta de Catalunya. Email: privera_vargas@uoc.edu

**Profesor Titular. Universitat de Barcelona. Email: cristina.alonso@ @ub.edu 


\section{Introducción}

El Elearning es una modalidad de enseñanza que desde hace dos décadas ha irrumpido con fuerza en el ámbito de la educación superior (Ferraté, 2011). Si bien su emergencia ha revolucionado la forma en que entendemos y ejecutamos los procesos de enseñanza y aprendizaje, este sigue estando en una fase de construcción y adecuación (Garrison y Anderson, 2005).

Dimensiones como el posicionamiento de la sociedad de la información, el uso y evolución de las Tics, junto a la emergencia de un marco postmoderno y reflexivo en el debate educativo (Lyotard, 1991; Giddens, 1993; Kuhn, 1996), son aspectos que en su conjunto están condicionando la dirección de las innovaciones educativas, entre las que se enmarca el desarrollo del Elearning.

En este expansivo escenario postmoderno y reflexivo (Giddens, Beck y Lash, 1997), identificamos dos aspectos del Elearning que tienen especial trascendencia. En primer lugar, su impacto y dinámica de ejecución, puede variar tanto por el modelo de desarrollo que se observe, como por el marco reflexivo y postmoderno en que se encuentre ${ }^{1}$ (Rivera, 2010). En segundo lugar, el Elearning responde a la necesidad actual de contar con modelos de enseñanza en educación superior, que sean capaces de adaptarse a las necesidades y los ritmos de vida de un nuevo tipo de estudiantado, que a la vez de ser más autónomo y protagonista de sus procesos formativos, cuenta con un manifiesto dominio en el uso de las Tics.

Esta segunda dimensión, no solo conforma el objetivo de este artículo, sino que también representan uno de los principales desafíos entre quienes buscan posicionar al Elearning, en el ámbito académico e institucional.

El presente trabajo está estructurado en seis partes. El primer apartado de introducción. En el segundo apartado, se presenta una descripción conceptual del marco social postmoderno y reflexivo, junto a las principales características de la sociedad de información. Finalmente, se analiza cómo estas dimensionas han condicionado el diseño y la actual dinámica de la educación superior. En el tercer apartado, exponemos las principales características del nuevo estudiantado en educación superior. En el cuarto apartado, a partir de la percepción de la comunidad académica (global y local) definimos la concepción actual del Elearning. En el quinto apartado, se presenta una discusión analítico - sociológica, en la que se confrontan los aspectos desarrollados a lo largo del artículo. Finalmente en las conclusiones, se pretende dar respuesta al objetivo de este trabajo, el cual se intenta desarrollar mediante el presente análisis bibliográfico. El objetivo planteado es el siguiente:

Establecer, de qué manera el actual escenario reflexivo, condiciona la emergencia de un nuevo estudiantado más activo y autónomo, y también del Elearning, como modalidad de enseñanza flexible.

Este trabajo, no tiene la pretensión de cerrar el foco analítico sobre este ámbito; por el contrario, al ser un ejercicio sustantivamente bibliográfico, busca promover la reflexión sociológica en el campo de la educación postmoderna y tecnológica, y a la vez incentivar el desarrollo de otros ejercicios analíticos y empíricos, que permitan dar una mayor consistencia al tema.

\section{Transformaciones sociales y cultu- rales en la sociedad contemporánea.}

En sus inicios, el proyecto de la modernidad consignó la importancia del conocimiento científico para el bienestar del ser humano, en tanto se constituye a sí mismo en esa actividad y ella le permite avanzar en el desarrollo de la humanidad. Pero justamente esta lógica es la que hoy en día empieza a cuestionarse, especialmente a la hora de determinar si aquellas promesas de mejora en la calidad de vida y bienestar de los humanos, se han cumplido o han quedado a la deriva.

Este nuevo marco analítico, ha implicado la emergencia de un pensar crítico y revelador, que sin embargo, aun no se sitúa como un pensar uniforme. De hecho, el concepto postmodernidad en sí mismo, solo nos sugeri-

1. Aspecto desarrollado por Rivera (2010). En el artículo, el autor sostiene que en las sociedades con mayor prevalencia del modelo de bienestar y a la vez actualmente reflexivas, el Elearning asoma como una alternativa académica que ha favorecido la inclusión social y la igualdad de oportunidades. Por el contrario, en sociedades periféricas, donde predomina un modelo social que otorga mayor prevalencia al mercado, el Elearning se ha posicionado como una alternativa de formación de alto costo, y a la vez selectiva y «compulsiva» (Giddens, Beck, Lash, 1997), lo que ha dejado de manifiesto, otro emergente fenómeno contemporáneo, «el credencialismo». 
ría la existencia de una era distinta a la moderna, una era posterior, contraria, pero no especifica respecto de «qué tipo de era». Un síntoma de la ausencia de un paradigma único que describa y explique adecuadamente la situación actual, es el debate en torno a la definición y estatus del propio concepto postmodernidad. Las hipótesis se mueven desde el extremo de considerarlo como la nueva época histórica cultural, que estaríamos gestando (y desde esa perspectiva la época moderna habría dado pie a la época postmoderna) hasta quienes lo reducen a una simple nueva sensibilidad para realizar determinadas actividades de la vida humana, especialmente el arte. En rigor, el concepto tiene su origen en el ámbito estético, pero de ahí se ha desplazado y ha terminado por acuñarse designando las diversas transformaciones de la cultura, el conocimiento $y$, en definitiva, de la forma en que el ser humano construye su vida en la actualidad.

Este escenario, caracterizado por la existencia de una mayor cantidad de incertidumbres que de certezas, no ha establecido un camino manifiesto por el cual podamos orientarnos. Lo que sí ha sido posible es la emergente resignificación de los principales símbolos del proyecto de la modernidad (Giddens, Beck y Lash, 1997).

\subsection{Postmodernidad y modernización reflexiva}

La modernidad reflexiva a la que aluden A. Giddens, U. Beck y S. Lash (1997), describe un fenómeno característico de aquellas sociedades que asimilaron el proyecto de la modernidad como base de sus propias posibilidades de progreso, y que en el presente, más que confianza, encuentran incertidumbre y la manifestación de permanentes riesgos sociales. Respecto de los riesgos, su proliferación, constituye un escenario social que antecede a la modernización reflexiva, y que Beck (1998) denomina como Sociedad del Riesgo?

La Sociedad del Riesgo indicaría justamente que los efectos de una naturaleza independiente de las actividades de las sociedades, son en realidad inexistentes, «no hay consecuencias que no la involucren (a la sociedad) y en donde sus sistemas organizativos no jueguen un rol decisivo». (Beck, 1998: 76).

En este marco de proliferación de riesgos sociales, y de falta de control de los sistemas organizativos, emerge la modernización reflexiva. Fenómeno, que por un lado, hace referencia a una época de la modernidad que se desvanece y, por otro, al surgimiento anónimo de otro lapso histórico, que no se gesta a causa de elecciones políticas, del derrocamiento de gobierno alguno o por medio de una revolución, sino que obedece a los efectos colaterales latentes en el proceso de modernización autónoma, según el esquema de la sociedad industrial occidental o mejor dicho «capitalismo». Para Kuhn (1996), el sujeto de esta destrucción creadora no es la crisis, sino el triunfo del orden a partir del proyecto científico de la modernidad.

La modernización reflexiva básicamente cuestiona la rigidez y la insuperabilidad de los supuestos de la sociedad industrial. No tiende a la autodestrucción, sino a la «auto trasformación» de la modernización industrial. No alude a la reflexión como el adjetivo «reflexivo» parece sugerir, sino más bien como una fase de auto confrontación: «el tránsito de la época industrial a la del riesgo se realiza anónima e imperceptiblemente en el curso de la modernización autónoma conforme al modelo de efectos colaterales latentes». (Beck, 1998: 120).

La modernidad reflexiva se puede manifestar con la crisis de la familia nuclear y la auto organización concomitante de las narraciones vitales, cuando se pierde fe en los beneficios que traería vivir en comunidades; con la pérdida de influencia de las estructuras de clase sobre los agentes: ${ }^{3}$ en la conducta electoral, en las pautas de consumo, en la afiliación sindical; con el desplazamiento de la producción regulada por la flexibilidad laboral, que desde luego trae consigo una baja en la calidad de vida de los trabajadores y un constante empobrecimiento; con la nueva desconfianza ecológica y la práctica de la ciencia institucionalizada; y con el cuestionamiento a los patrones y modelos educativos tradicionales, que reproducen una sociedad que ya no genera confianza.

2. El principio axial de las sociedades del riesgo son los peligros generados por la civilización moderna, los cuales ya no pueden ser ni temporal, espacial o socialmente delimitados, de tal forma que los fundamentos de la sociedad industrial (las instituciones elementales tales como el estado nación, los procesos fundamentales como los antagonismos de clase, las visiones del control y de la racionalidad técnico-económicas y sobretodo la independencia entre la tecnología y la política) son socavados, superados o eludidos sistemáticamente (Beck, 1998).

3. Representan a entes de la sociedad que permiten la socialización, como la familia, los medios de comunicación, los grupos de pares, etc. Giddens (1993). 


\subsection{La Sociedad de la información: cambios en la construcción del saber y emergencia de las TICS}

En términos generales podemos llamar Sociedad o Era de la Información «a la utilización masiva de herramientas electrónicas con fines de producción, intercambio y comunicación.» (Castells, 2001:398).

En este punto, la sociedad de la información obedece a la utilización masiva de herramientas electrónicas para el intercambio comunicacional de las personas, donde su masificación denominativa las señala como tecnologías de la información y la comunicación social (Tics). Así, hoy asistimos a la emergencia de la industria de la informática en la creación, distribución y manipulación de los saberes y productos culturales, en la renovación y revolución de los procesos de enseñanza y aprendizaje (Castells, 2001; Cabero et al., 2008; Sangrà et al., 2011)4 .

Es relevante señalar que la generación, procesamiento y transmisión de la información se convierten en fuentes de poder, por este motivo, resulta primordial focalizar la atención en el mantenimiento de la brecha digital y en la exclusión de los países más pobres, al concentrarse la riqueza en solo en determinadas sociedades. Hay que tener presente además, que existen visiones críticas que emergen desde múltiples escenarios académicos y sociales. En ellas se señala que esta llamada «Sociedad de la Información» no es sino una versión actualizada del imperialismo cultural ejercido desde los países ricos hacia los pobres, especialmente porque se favorecen esquemas de dependencia tecnológica ${ }^{5}$.

\section{Las Tics en los procesos de enseñanza y aprendizaje}

El posicionamiento de la sociedad de la información, impulsado tanto por el avance científico, como por el uso generalizado de las tecnologías de la información y la comunicación (Tics), ha conllevado cambios que han alcanzado todos los ámbitos de la actividad humana. Sus efectos, podemos identificarlos de manera explícita en los ámbitos político y económico, pero sobre todo en la confección de los sistemas educativos contemporáneos (Sancho, 1998). Esto se evidencia tanto en la razón de ser de las instituciones educativas, como también en la formación básica que precisamos, es decir, la forma de enseñar y de aprender, junto a las infraestructuras y los medios que utilizamos para ello (Castells, 1997).

Para Adell (1997), se haría así imprescindible considerar en este proceso a las Tics como herramientas fundamentales para la generación del saber, más aún, cuando se trata de diseñar los sistemas educativos contemporáneos.

Gros y Lara (2009) sostienen, respecto del uso de las Tics en los procesos formativos, que éstas, al ya existir y estar a disposición de la ciudadanía, deben ser utilizadas para fomentar y facilitar los procesos de creatividad e innovación por parte del estudiantado. Para las autoras, la consolidación del proceso de cambio en la forma de gestionar el conocimiento y por ende, de construir sistemas educativos, se encuentra en el presente, indisociablemente vinculada a la innovación (Gros y Lara, 2009).

La innovación, así concebida, surge como un elemento de creación de nuevos conocimientos, productos y procesos, siendo obligatoria su consolidación en la vida de las organizaciones (Gros y Lara, 2009). A su vez, resulta ser un mecanismo de diferenciación estratégica, es decir, «una parte indiscutible de la cartera de valores del siglo XXI» (Gros y Lara, 2009: 25).

Por tanto, la implementación de las Tics en los sistemas educativos contemporáneos, y la necesidad de una política educativa que favorezca la innovación y que a la vez otorgue relevancia a las actuales formas de construir el saber de los individuos, (tanto en lo cotidiano como en lo formal), requiere de instituciones con dinámicas flexibles y sobre todo contextualizadas en la realidad, que den cabida y que permitan el desenvolvimiento tanto de «Inmigrantes digitales» como de «Nativos digitales» (Prensky, 2001).

Esto último es de vital consideración, ya que de acuerdo a lo señalado por Casablancas et al. (2006), los

4. Esto se pudo evidenciar en los acuerdos finales de la Cumbre Mundial de la Sociedad de la Información (CMSI) realizada en Ginebra en el año 2003, donde se manifestó que la comunicación y la educación mediada por Tics, puede otorgar instrumentos para la función crítica y educativa, en pro de satisfacer las demandas de una nueva ciudadanía (CMSI, 2003).

5. Podemos citar a Marcuse (pre sociedad de la información) en «El hombre unidimensional» (1968). Y en reflexiones más actuales, a Beck (1998), Giddens (1993). 
modelos mentales cambian lentamente, y necesitan cierto tiempo de maduración para que se puedan considerar aprendizajes profundos. En el caso de la incorporación de tecnologías en el ámbito de la educación, el tiempo de maduración conlleva un lapsus de conocimiento respecto a «qué son» las tecnologías y «qué pueden» aportar a la educación desde la perspectiva de la enseñanza y desde la perspectiva del aprendizaje (Casablancas et al., 2006). Más aún de las formas de generación del conocimiento en sí mismo.

A partir de la importancia del actual marco de profesionalización social, así como de la mayor relevancia que cada día tiene la formación permanente de las personas, emerge un nuevo estudiantado, que busca tener cabida para satisfacer las nuevas necesidades funcionales de la sociedad por reproducirse. A la vez, resulta de especial importancia observar cómo las instituciones de educación superior han enfrentado estos cambios, y cómo han pasado de ser instituciones rígidas, a ser instituciones flexibles en la búsqueda de una actualización de los nuevos procesos de gestión educativa.

A continuación presentamos algunas de las principales características de este nuevo estudiantado, que busca desprenderse de los modelos formativos tradicionales, y que a la vez espera ser protagonista de los procesos de enseñanza/aprendizaje en los que se involucra.

\section{La emergencia del nuevo estudian- tado y la innovación educativa}

Los expertos enfatizan que para dar respuesta a las necesidades educativas actuales es necesario modificar el papel que desempeñan los agentes implicados en el contexto educativo, esto es, replantear el espacio educativo y desempeñar nuevos roles docentes y discentes (Lynch, 2002; Majó y Marquès, 2002; Romeu, 2011).

De manera general, el acceso por parte de los estudiantes y los docentes a la inagotable fuente de información que propician las TIC, abre un sinfín de posibilidades educativas (Majó y Marquès, 2002), rompiendo el clásico paradigma de formación tradicional característico del proyecto educativo existente en aquellas sociedades organizadas en búsqueda de la modernidad (Giddens, 1993), de la clase magistral centrada en el docente, donde el alumnado adoptaba un papel pasivo y repetitivo (Majò y Marquès, 2002).
Este nuevo estudiantado es resultado de su época, de la misma forma que otros tipos de estudiante a lo largo de la historia de la humanidad, han sido el resultado de otras situaciones y otras necesidades.

En el presente, el rápido desarrollo de las tecnologías de gestión y comunicación, así como la competitividad, han obligado a una dinámica de aprendizaje e incorporación mas continúa. La necesidad permanente de formación ha generado un nuevo alumnado diferente al tradicional (Borges, 2007; Moreno, 2009), con demandas propias. En este grupo se encuentran personas que, por determinadas situaciones, no pueden acceder a los centros de aprendizaje con modelos presenciales convencionales. Además, el nuevo alumnado precisa de conocimientos y habilidades específicas que sean inmediatamente aplicables a su potencial área profesional, y que integren sus conocimientos y habilidades previamente adquiridos (Marqués, 2008).

Ante este escenario, las Tics desempeñan un rol determinante, toda vez que han puesto, una gran variedad de nuevos materiales a disposición, tanto para el alumnado, como para el profesorado. La posibilidad de emplear los diferentes recursos audiovisuales y sobre todo, la facilidad de comunicación y fuente de información que supone Internet, han abierto un vasto campo para la formación.

Los cambios en los estudiantes y en los materiales, han variado la concepción de la enseñanza y el aprendizaje. Autores como Pallof y Kratt (2003) y Moreno (2009), desde una visión constructivista, sostienen que el proceso de aprendizaje con Tics, es básicamente colaborativo: Ios estudiantes crean conocimiento a través de la interacción entre ellos mismos, con el profesor, y con su entorno. El alumnado, así deja de tener un papel meramente pasivo en su proceso de aprendizaje (Moreno, 2009).

\subsection{El estudiantado virtual: aprender en Entornos Virtuales de Aprendizaje (EVA)}

La clasificación de Prensky (2001), respecto a la existencia de nativos analógicos, inmigrantes digitales y nacidos digitales (Prensky, 2001; 2006), podría ser la clave para intentar comprender las actuales transformaciones en la configuración de los sistemas educativos contemporáneos.

La gran mayoría de la primera oleada de estudiantes de EVA (finales del siglo XX y comienzos del siglo XXI) tomó contacto con el ordenador y con las TIC en la juven- 
tud tardía 0 en la vida adulta, es decir, son inmigrantes digitales, provenientes de un desempeño formativo en el que el profesor era el centro del hecho educativo, quien establecía los ritmos, cómo y hasta dónde se debía adquirir conocimientos.

Estos estudiantes, con carencias en las destrezas y competencias para aprender en un EVA, suelen comenzar sus estudios en un entorno virtual convencidos de que habrá un profesor esperándolos, dispuesto a transmitir su conocimiento de forma telemática. Cuando descubren que buena parte del aprendizaje proviene de la participación en el aula, de la colaboración con los compañeros y de profundizar uno mismo en los contenidos a partir del material de estudio, pueden sentirse desilusionados 0 incluso «estafados». Como explican Palloff y Pratt (2001: 108), es el descubrimiento de que el profesor no es «el experto en el estrado», sino "el guía que acompaña», cuya misión es ayudar al estudiante en el aprendizaje que realiza, y que nadie puede llevarlo a cabo sino es él (o ella). A la vez fomenta la interacción y la colaboración entre el propio estudiantado. «Es este un cambio importante, para el cual, los estudiantes no deberían encontrarse solos, sino debidamente preparados por sus profesores 0 por su institución.» (Borges, 2007: 5).

Las siguientes oleadas de estudiantes virtuales están formadas por estudiantes de una gran variedad de edades, entre los que paulatinamente serán mayoría los nacidos digitales, aún provenientes de una formación reglada centrada en la transmisión de conocimientos realizada por el profesor, pero más proclives a la proactividad, a la colaboración entre iguales, a relaciones telemáticas más democráticas y menos jerárquicas ${ }^{6}$.

Para desempeñarse adecuadamente en un entorno virtual, los estudiantes tienen que ser competentes en una serie de acciones y de actitudes (Flores, 2004); por ejemplo, en escribir de forma adecuada y organizada, en la lectura extensiva, en comunicarse por medio del correo electrónico, en el manejo del entorno virtual y sus herramientas, en la búsqueda, selección y difusión de información (Jiménez, 1999), en organizar el tiempo de estudio y de conexión, en relacionarse adecuadamente con otros compañeros, organizando el trabajo común, aportando, debatiendo y discrepando.
Aunque existen lógicas diferencias y necesidades, entre estudiantes virtuales según el área 0 titulación, éstos muestran características comunes en su identidad y en su desempeño al aprender en un EVA (Borges, et al., 2007). Quizá la coincidencia más llamativa sea que la mayoría se incorpora a la formación en un EVA sin saber en qué consiste ser un estudiante virtual, sin saber qué hay que hacer, qué comporta, cómo desempeñarse óptimamente, sin haber recibido formación al respecto, (Borges, 2007).

En este sentido, según Borges (2007), el estudiante virtual, en el contexto de la educación superior, se encuentra frecuentemente en una organización virtual, aspecto que implica un mayor uso de las TIC, unos materiales de aprendizaje con una base tecnológica mayor, un cambio en la interacción social y un desarrollo diferente de las actividades de aprendizaje (Harasim et al., 2000). Así, basa su formación en la flexibilidad, disponiendo individualmente de su tiempo bajo su responsabilidad (haciendo compatible la actividad formativa con la laboral y personal) y accediendo a todos los elementos del proceso educativo de manera permanente.

Respecto de la modalidad de enseñanza mediada por Tics, en el que se desenvuelve preferentemente este nuevo estudiantado virtual, existen muchas definiciones. Entre ellas: Computer Based Learning (CBL) (Paulsen, et al., 2003), Virtual Learning (Morgan, 2003) o Virtual Education (Ahmad, 2010). En el contexto iberoamericano, se usan conceptos como Formación virtual, Aprendizaje Electrónico, Educación On line, Aprendizaje Virtual, (Cabero, 2008; Area y Adell, 2010; Moreno 2011). Sin embargo, todos son términos que, con ciertos matices, sostienen una idea similar: proceso de enseñanza y aprendizaje a través del uso de las nuevas tecnologías. Para este trabajo con el fin de presentar un concepto universalmente reconocido que articule posturas y definiciones, llamaremos a esta modalidad de enseñanza, Elearning.

\section{El Elearning y los nuevos procesos de enseñanza en educación superior}

El Elearning es una modalidad de formación y aprendizaje en educación superior que ha derivado (Simonson,

6. No obstante, el hecho de haber nacido y crecido habituados a las TIC no convierte automáticamente a los nacidos digitales en estudiantes proactivos, colaborativos, autónomos y participativos (Borges, 2007), por lo que las destrezas y las competencias relacionadas con el desempeño en un EVA se tendrían que trabajar como parte del currículo transversal. 
et al., 2006) o ha estado inspirada (Sangrà, et al., 2011) en la tradicional concepción de educación a distancia. Además, como se ha indicado anteriormente, su emergencia y evolución han estado condicionadas a la inserción y utilización del Tics en el ámbito educativo.

Respecto de la búsqueda de una definición general, podemos evidenciar, en diversos trabajos académicos existentes (Gisbert, 2002; Cabero, 2006; Simonson, et al., 2006) que concurren cinco características singulares:

- Se trata de una modalidad moderna de la tradicional enseñanza a distancia.

- Consiste en un proceso de educación formal, que posee una base institucional.

- Se expresa en la separación física del profesorado y el alumnado.

- Utiliza sistemas de telecomunicación interactivos.

- Conecta al alumno y al profesor con los recursos de aprendizaje, compartiendo información y datos, entre otros elementos, por medio de distintos formatos que integran la experiencia educativa.

La particularidad de esta modalidad radicaría tanto en la no co-presencialidad entre el educador y el educando, como en el hecho de que todos o la mayor parte de los contenidos son facilitados online. Según Allen y Seaman (2009) se habla de un modelo Elearning cuando más de un $80 \%$ de dichos contenidos son entregados a distancia, y donde (comúnmente) no se contemplan encuentros cara a cara. Esta separación física se ve compensada con mecanismos de vinculación de las partes involucradas entre sí y con los contenidos definidos por medio de la utilización de modelos pedagógicos y tecnológicos acordes. «Los sistemas de telecomunicación permiten la interacción mediante actividades sincrónicas y asincrónicas que posibilitan la generación de un espacio educativo eficaz a distancia.» (Mendiburo y Reininger, 2011: 104-105).

Posiblemente, uno de los trabajos sobre Elearning más significativos en el concierto global sea el realizado por D. Randy Garrison y Terry Anderson (2005). Se trata de un ejercicio que buscaba ser una guía para la investigación y práctica del Elearning en vistas a promover una educación superior de alto nivel (Garrison y
Anderson, 2005: 27). Para los autores, el Elearning representa una modalidad capaz de crear comunidades simultáneas y accesibles para todos los actores en cualquier momento y lugar (tanto de investigación, como de docentes y estudiantes).

Respecto a su trascendencia, para Garrison y Anderson (2005), el Elearning con una pedagogía adecuada y un plan docente mediato, podrá transformar la educación superior (Garrison y Anderson, 2005). «Si bien el Elearning es una maravillosa fuente de ideas en el marco de la sociedad de la información, para ser genuinamente educativa deben ofrecer una experiencia que asegure continuidad o la base de experiencias educativas nuevas y valiosas». (Garrison y Anderson, 2005: 31). Por tanto, debe verse como una modalidad que va mucho más allá de ser considerada una herramienta de soporte al desarrollo de las clases, «en la medida que todas sus potencialidades se conozcan y asimilen en las distintas instituciones educativas, podremos presenciar una transformación educativa sin precedentes.» (Garrison y Anderson, 2005: 18).

Para Garrison y Anderson (2005), el Elearning siempre ha buscado (desde su origen formal establecido en el año 1996), la adaptación a los sistemas educativos contemporáneos, facilitar el acceso y cobertura de la educación superior, y la búsqueda de los estándares de calidad pertinentes. Estos aspectos representan en el presente y representarán en el futuro, los principales desafíos del Elearning (Garrison y Anderson, 2005).

\subsection{Concepciones del Elearning en España}

En el contexto académico español, existen múltiples esfuerzos conceptuales e investigativos que han buscado construir una definición integradora del Elearning. Manuel Area (2012) por ejemplo, sostiene que la emergencia del Elearning ha estado marcada por el hecho de que las instituciones educativas han tomado conciencia de que no pueden dar la espalda a la realidad tecnológica y por ello han empezado a desarrollar políticas destinadas a crear las condiciones necesarias para que las Tics estén presentes en las actividades formativas. El autor hace referencia a las políticas desarrolladas por las propias universidades, que estimuladas desde la CRUE (Conferencia de Rectores de las Universidades Españolas) han facilitado que los campus virtuales sean una realidad manifiesta en casi todas universidades. 
Para Area (2012), el Elearning no es solo una modalidad que consiste en añadir las Tics a los procesos de enseñanza y aprendizaje tradicionales. Se trata de un cambio educativo mucho más profundo, que supone la reinvención de los procesos educativos, convirtiéndolos en una nueva forma de acceder a la educación, promoviendo un mayor protagonismo del sujeto que aprende (personalización). «En el marco del Elearning, se produce una gran riqueza comunicativa y una manifiesta interacción, entre el estudiantado (comunicación social), por lo que el aprendizaje incidental o informal se entremezcla, y a veces se confunde con los procesos formativos formalizados.» (Area, 2012: 5).

En la misma línea, para Julio Cabero (2008), el Elearning es un entorno formativo que posee tres características básicas: (1) es una formación a distancia, que está basada en el uso de las Tics y que son apoyadas por Internet; (2) se trata de un proceso de enseñanza-aprendizaje que se lleva a cabo a través de Internet, caracterizado por una separación física entre profesorado y estudiantes, pero con el predominio de una comunicación tanto síncrona como asíncrona, a través de la cual se lleva a cabo una interacción didáctica continuada y (3) «el alumno pasa a ser el centro de la formación, al tener que auto gestionar su aprendizaje, con ayuda de tutores y compañeros.» (Cabero, 2008: 40).

En el año 2011, el eLearn Center ${ }^{7}$ de la Universitat Oberta de Catalunya, realizó una investigación que buscó significar y dar consistencia a la definición del Elearning. Se trató de una investigación dirigida por Albert Sangrà, cuyo propósito fue construir una definición del Elearning que pudiese ser aceptada por la mayor parte de la comunidad científica y que a la vez sirviera como referente para los estudiosos y profesionales de esta temática.

Según lo expresado en el informe final de la investigación, (Sangrà et al., 2011), el Elearning es una modalidad que va mucho más allá del uso de las tecnologías. «Las características predominantes del concepto hacen referencia a la modalidad de enseñanza y aprendizaje, a un nuevo modelo educativo, pero también la explotación de medios y dispositivos electrónicos.» (Sangrà, et al., 2011:
5). Paralelamente se destacan algunos atributos, como la flexibilidad o la interacción, y se hace hincapié en el rol activo de los estudiantes y del profesorado.

Para los autores del informe, el Elearning «Se trata de una modalidad de enseñanza y aprendizaje que puede representar todo o bien una parte del modelo educativo en el que se aplica, y que explota los medios y dispositivos electrónicos para facilitar el acceso, la evolución y la mejora de la calidad de la educación y la formación.» (Sangrà, et al., 2011: 6).

\section{Discusión Analítica: ell e-Learning como respuesta reflexiva a los proce- sos de enseñanza formal en educación superior}

Como hemos expresado a lo largo de este trabajo, no existe en el presente un criterio externo 0 interno de carácter absoluto, que permita medir el avance y consolidación del proyecto de la modernidad (Lyotard, 1991). Más bien lo que identificamos, es un marco de incertidumbre que afecta a casi todas las dimensiones que constituyen la dinámica social.

Este estado de incertidumbre ha estado representado por la emergencia de un sentir postmoderno que ha evidenciado como el proyecto de la modernidad, terminó traicionándose a sí mismo, debido a que el elemento emancipador y liberador que contenía dicho esfuerzo, terminó convirtiéndose en un «totalitarismo de la razón», al absolutizar el camino único: el método (Kuhn, 1996). De ahí que la liberación sea entendida hoy como el despliegue de lo distinto, de las diferencias en los más distintos ámbitos de la vida cultural y cognoscitiva del ser humano. En este marco postmoderno, y reflexivo (Giddens, Beck y Lash, 1997), las formas en que se han diseñado los modelos de enseñanza y aprendizaje, tienden a ser revisados y diversificados ${ }^{8}$.

La modernidad reflexiva se puede evidenciar con la pérdida de la fe en las promesas de un mundo moderno y equilibrado, en la práctica de la ciencia institucionalizada;

7. Centro de investigación y desarrollo en Elearning, de la UOC. http://www.uoc.edu/portal/ca/elearncenter/index.html

8. Además, emerge el fenómeno de la individualización (Rivera, 2008), que ha liberado a los individuos de las estructuras colectivas y abstractas, tales como la clase, la nación, la familia nuclear, la educación tradicional, y la creencia incondicional en la validez de la ciencia. Esta individualización se manifiesta tanto en conductas como en expectativas, las cuales no pueden ser satisfechas en el escenario social formado bajo el amparo del proyecto de la modernidad. 
y desde luego, en los patrones y modelos educativos que reproducen una sociedad que ya no genera confianza. En este sentido, para Beck (1998), las instituciones educativas (escuelas y universidades) en el marco de la modernidad, se convierten en «estaciones fantasmas», un lugar donde es preciso acudir, pero que expide credenciales para ninguna parte. Para Beck, el marco de enseñanza moderno, no considera la emergencia de nuevas identidades sociales, las cuales si bien siguen acudiendo a los centros formativos, lo hacen como una práctica compulsiva y repetitiva. Solo fenómenos reflexivos, como el aumento manifiesto del paro, han logrado cuestionar este orden normativo (Beck, 1998).

Según Beck (1998), el problema se sustenta en la falta de garantías de empleabilidad que entrega la formación profesional. Por tanto, los itinerarios instructivos orientados a la profesión, cuando su futuro profesional se torna más oscuro, modifican esencialmente su sentido, aunque las instituciones formativas no se flexibilicen o incluso sigan manteniendo sus contenidos curriculares. «Ello puede permanecer escondido para los planificadores e investigadores de la educación, en el gran saco de las «discrepancias entre formación y ocupación», e incluso puede permanecer escondido para los docentes, pero no para el nuevo estudiantado que más tarde, al dejar el sistema educativo, encuentra las puertas cerradas del sistema de empleo (principalmente por falta de asimilación de las competencias profesionales adecuadas, y por la nula experiencia laboral previa), lo que anticipan de manera autoconsciente mientras están en la instrucción» (Beck, 1998: 238). Es decir, que por la irrupción externa del mercado de trabajo, son dañados o destruidos los fundamentos del carácter inmanentemente formativo de la instrucción con referencia ocupacional. Lo que en el marco de la preponderancia del mercado en la construcción de la sociedad, debería hacer replantear el sentido rígido de las instituciones formativas.

Para Rafel Feito (2009), el problema también estaría dado por el hecho de que en el marco moderno, la búsqueda de legitimidad institucional, está por sobre la búsqueda de legitimidad del proyecto educativo y de su sentido. «Una buena formación académica no es, por sí sola, garantía para afrontar con éxito los retos que habremos de afrontar en los diversos escenarios que conforman nuestra vida adulta» (Feito, 2009).

Esta imagen de la «estación fantasma» resulta equivalente a la de la «institución zombie», que se deriva de la opinión de Beck (1998), a propósito de las instituciones sociales que se mantienen de manera inerte aunque haya concluido su ciclo vital (instituciones sociales «zombies»). En el campo de la educación superior, la imagen de la Universidad «zombie» tiene además, la potencia de ilustrar la situación de desconcierto en que se haya buena parte del sistema educativo, derivado en gran medida de «el virtual encapsulamiento del sistema educativo respecto de cualquier mensaje del mundo exterior». Por tanto, pensar en la universidad como una institución dinámica y activa, fuera de los cánones convencionales y más cerca de los ritmos de vida del nuevo estudiantado, sería el resultado de un proceso reflexivo, y no necesariamente una acción inherente a la prevalencia del mercado, o a la propia configuración educativa del proyecto moderno (Aránguiz y Rivera, 2011).

Hoy en día, existen muchas iniciativas que buscan flexibilizar la rigidez de las instituciones educativas. Ya sea favoreciendo la formación continua o permanente 0 haciendo compatible la acción educativa con el ritmo de vida personal del estudiantado, rescatando sus saberes propios, la mayoría de los cuales son asimilados en la cotidianeidad (Aránguiz y Rivera, 2011).

Respecto de la necesidad de favorecer estos saberes cotidianos, para Hernández (2004), una de las mayores transformaciones de la globalización consiste en construir y reconstruir la sociedad del conocimiento y de la cultura; es decir, prolongar, y no reducir la formación; desligarla o separarla de puestos de trabajo y oficios concretos. «El hecho de orientar los procesos educativos hacia cualificaciones clave que respondan a esa perspectiva no se ha de concebir sólo como «flexibilidad» ni como «formación continuada», sino que también representa situar la formación en contextos de competencia social, capacidad de dirección, habilidad ante conflictos, comprensión cultural, mentalidad de relación y acceso a las inseguridades y paradojas de la segunda modernidad.» (Hernández, 2004: 2).

Respecto a la importancia creciente por la educación informal y cotidiana de las personas, Kahl (1997), sostiene que un aspecto explícito en la forma de diseñar la gestión pedagógica, debe ser capaz de realzar el contexto cotidiano de las personas, y por ende, sus saberes adheridos a esta realidad. «Hay que vincular el aprendizaje con hechos cotidianos de modo que las personas puedan dominar su vida.» (Kahl 1997, en Beck 1999: 101). 
Ambos aspectos de flexibilización de la enseñanza, se ven favorecidos a partir de la llegada de las Tics al campo de la educación. Para Castells (1997), esto se debe también a la legitimidad y omnipresencia de las Tics y los medios de comunicación social en los aprendizajes que las personas realizamos informalmente a través de nuestras relaciones sociales, y que por lo mismo, cada vez tienen más relevancia en nuestro bagaje cultural.

De igual forma, ni incorporar de manera compulsiva (Giddens, 1993; Beck, 1998) a las Tics en la generación de conocimiento y en la conformación de los sistemas educativos contemporáneos, ni rechazar su potencialidad y aplicabilidad, parece ser el camino. Para Adell (1997) y Sancho (1998), no se trata de construir un nuevo modelo formativo solo en función del potencial de las Tics o por el contrario, su rechazo y resistencia. En tal sentido para Sancho (1998), las posturas tecnófobas como las tecnófilas, no solucionan las problemáticas postmodernas en los sistemas educativos ${ }^{9}$. Para la autora, es indispensable tener presente una perspectiva histórica, social, cultural y política, tanto de la tecnología como del contexto social, antes de involucrar a las TICS en los procesos de enseñanza contemporáneos.

En la misma dirección Gros y Lara (2009) sostienen que más allá del debate sobre el uso de las Tics en los procesos formativos, lo cierto es que éstas, al ya existir y estar a disposición de la ciudadanía, deben ser utilizadas para fomentar y facilitar los procesos de creatividad e innovación por parte del estudiantado.

En este sentido es que el Elearning emerge como una posibilidad de articular todas estas necesidades de cambios en los modelos de enseñanza. De transformar a la universidad en una organización activa, acorde a los tiempos y demandas contemporáneas, que pueda dar cabida a quienes desde este marco emergente de la individualización, buscan obtener un conocimiento práctico y funcional, y que a la vez refuerce sus saberes cotidianos.

Por tanto, en la línea de lo planteado tanto por Garrison y Anderson (2005), como por Area (2012),
Cabero, et al., (2008) y Sangrà, et al., (2011), consideramos que el Elearning es una modalidad de enseñanza, que si bien ha emergido como evolución de la educación a distancia tradicional, es a la vez el producto de diferentes procesos sociales marcados por la consolidación de la sociedad de la información y el desarrollo y preeminencia de las Tics en la construcción del conocimiento contemporáneo. El Elearning sería el resultado de los procesos de avance y mejora de la educación superior y de distintos esfuerzos académicos realizados para facilitar el acceso y mejorar la calidad de los actuales procesos formativos. Todo lo anterior, inspirado por el marco reflexivo al que hemos hecho referencia, que pone en jaque los procesos convencionales de formación promovidos en el desarrollo proyecto de la modernidad que reproducen una sociedad que ya no genera confianza y que no son coherentes ni adaptables al marco activo en el que se desenvuelve este nuevo estudiantado

\section{Conclusiones}

Tal como hemos manifestado en la introducción, esta aportación es el resultado de un ejercicio analítico, cuya pretensión ha sido establecer, a partir de una revisión bibliográfica, de qué manera el actual escenario reflexivo que viven las sociedades contemporáneas, ha condicionado la emergencia de un nuevo estudiantado y, consecuentemente, del Elearning.

En tal sentido, podemos manifestar que en el marco del cuestionamiento estructural del proyecto en búsqueda de la modernidad, que ha caracterizado a las sociedades occidentales, en el ámbito de la educación superior, se ha evidenciado la necesidad de modificar el sentido de las instituciones educativas, debido a que tienden a no ser representativas de las necesidades reproductivas de la sociedad, ni de los ritmos y dinámicas del estudiantado actual. En este contexto toma sentido la emergencia de una universidad postmoderna, esencialmente crítica con la rigidez que tuvo durante años, y que busca ser un espacio de generación de conocimiento válido y actualizado.

9. Para Sancho (1998), la postura tecnófoba olvida que rechazando la consideración de cualquier variación en el trabajo docente, está utilizando mecánicamente un conocimiento tecnológico que acepta y reproduce sin reflexión, convirtiéndola en una técnica fosilizada que no tiene en cuenta las variaciones del contexto en el cual se está aplicando. Mientras la postura tecnófila, solo considera «tecnología» a las máquinas y aparatos y al conocimiento elaborado desde ámbitos que tienen poco que ver con los problemas a los que ha de responder la educación, desconsiderando el conocimiento práctico y teórico acumulado por años de estudio y experiencia. (Sancho, 1998: 15) 
El Elearning, es una modalidad de enseñanza en educación superior, mediada por las Tics, que, entre otras cosas, promueve la dinámica de la formación permanente y la consideración de los saberes propios del estudiantado. Se trata de una respuesta que emerge en el marco de la modernización reflexiva, y que por tanto busca mejorar y actualizar los procesos de asimilación de saberes y contenidos necesarios para reducir la incertidumbre y para promover la generación de confianza social y colectiva entre los distintos actores sociales.

\section{Referencias Bibliográficas}

Ahmad, Z. Virtual Education System (Current Myth \& Future Reality in Pakistan). Virtual University of Pakistan, November 16, 2010. Entrepreneurial Tutors. [Fecha de consulta: 30/02/2013]. Disponible en: http://papers.ssrn. $\mathrm{com} /$ sol3/papers.cfm?abstract_id $=1709878$

Aránguiz C. y Rivera P. «Desafíos de la universidad en la formación del siglo XXI: Construcción de ciudadanía crítica y activa». Edit. Sistemas de información. Castellón, España. [Fecha de consulta: 26/02/2012]. (2011). Disponible en: http://www.quadernsdigitals.net/index. php? accionMenu=hemeroteca. VisualizaArticulolU.visualiza \&articulo_id=11039\&PHPSESSID=2d79999838a38f990f8a $4 d 470 \mathrm{cc} 88189$.

Área, M. La formación y el aprendizaje en entornos virtuales. Potencialidades, debilidades y tendencias. Crítica. Noviembre-Diciembre, Núm. 982(2012):33-36.

Beck, U. «La Sociedad del Riesgo. Hacia una nueva modernidad». Paidós, Barcelona. (1998):237-238.

Borges, F. El estudiante de entornos virtuales. Una primera aproximación. En: Federico BORGES (coord.). El estudiante de entornos virtuales [dossier en línea]. 2007. Digithum. No 9. UOC. [Fecha de consulta: 30/02/2012]. Disponible en http://www.uoc.edu/digithum/g/dt/esp/ borges.pdf

Cabero, J. et al. «e-Learning: Meta-análisis de investigaciones y los resultados alcanzados». Proyecto de investigación. EA2007: 0326. Años 2007-2008. Investigador responsable: Julio Cabero A. Universidad de Sevilla. [Fecha de consulta: 26/11/2012]. Disponible en: http://tecno logiaedu.us.es/tecnoedu/images/stories/ ProyectoEA08.pdf
Casablancas, S.; Duran, P.; Alonso, C. e Higueras, E. Una experiencia de tecnología educativa relatada desde otros tiempos y espacios, Revista Latinoamericana de Tecnología Educativa. 6(1)(2007):33-47. [Fecha de consulta: 30/06/2012]. Disponible en http://campus virtual.unex.es/ cala/editio/

Castells, M. La era de la información. Economía, sociedad y cultura. Vol. 1 La sociedad red. Madrid: Alianza, 1997.

Castells, M. La galaxia internet. Reflexiones sobre internet, empresa y sociedad. Editorial Areté. Barcelona, 2001.

Comte A. «Curso de Filosofía Positiva». Paris, Francia. (1842):25-26.

CMSI. «Declaración de Principios», Ginebra. 2003. [En línea] Disponible en: http://www.itu.int/dms_pub/itu-s/ $\mathrm{md} / 03 /$ wsis/doc/S03-WSIS-DOC-0004!!PDF-S.pdf [Consultada: 11/03/2010 ]

Ferrater, G. A bunch of geniuses. Walk In Review n ${ }^{0} 7$. Edit SyL UOC. Barcelona: Universitat Oberta de Catalunya. (2011):22-39.

Feito, R. Otra Escuela es Posible. Madrid, España: Edit Siglo XXI, 2009.

Flores, J. How to Become a Proficient Online Learner. EE.UU.: Authorhouse - 1stBooks, 2004.

Garrison, D. R. y Anderson, T. El e-learning en el siglo XXI: Investigación y práctica. Octaedro. Barcelona, 2005.

Giddens, A. Consecuencias de la modernidad. Madrid: Alianza, 1993.

Giddens, Beck, Lash. «Modernización Reflexiva: Política, tradición y estética en el orden moderno». Madrid: Alianza, 1997.

Harasim, L.; Hiltz, S. R.; Turoff, M; Teles, L. Redes de aprendizaje. Guía para la enseñanza y el aprendizaje en red. Barcelona: Gedisa, 2000.

Hernández, F. «Educación y sociedad de riesgo» (abril 2004). Universidad de Valencia. (2004):2-4 
Jiménez, B. «Formación continua y nuevas tecnologías: una visión didáctico-comunicativa». En: J. Cabero Almenara [et al.] (Coord.) (1999). Las nuevas tecnologías para la mejora educativa. Nuevas tecnologías en la formación flexible y a distancia. Sevilla: Editorial Kronos, 1999.

Kahl, R. «Globalisierung zwingt zu einer Reorientierung des Lernens», en taz de (nota de Beck), 1997.

Kuhn T. «La Estructura de las Revoluciones científicas» Brevarios del Fondo de Cultura Económica. $1^{\text {a }}$ edición. México. (1996):121.

Lyotard J.F. «La condición postmoderna». Ediciones Cátedra. Madrid, 1991.

Marqués, P. Impacto de las TIC en la enseñanza universitaria. Didáctica, Innovación y Multimedia. Año 4. № 11. Barcelona, 2008.

Majó, J. y Marquès, P. La revolución educativa en la era internet. Barcelona: CissPraxis, 2002.

Morgan, G. Faculty Use of Course Management Systems, 2, retrieved November 27, 2005.

Moreno, P. Análisis del uso universitario de plataformas de gestión del aprendizaje. Estudio de caso en la Universitat de València. Tesis doctoral, Doctorado en Ingeniería Informática. Dirigida por Vicente Cerverón Lleó. Universidad de Valencia. (2009):27-29.

Paulsen, C., Et al. Usability test of an internet-based informatics tool for diabetes care providers: the comprehensive diabetes management program. 2003.

Palloff, R. M.; Pratt, K. Lessons from the Cyberspace Classroom. The Realities of Online Teaching. San Francisco, EE. UU.: Jossey-Bass. 2001.
Popper, K. Conjeturas y refutaciones: el desarrollo del conocimiento científico. Trad. Néstor Miguez (1994). Ediciones Paidós. Barcelona. 1994.

Prensky, M. «Digital Natives, Digital Immigrants Part 1». On the Horizon. 9(5), págs. 1-5. [Fecha de consulta: 22/05/2012]. Disponible en http://www. emeraldinsight. $\mathrm{com} /$ Insight/ViewContentServlet? Filename=/published/ emeraldfulltextarticle/pdf/2740090501.pdf. 2001.

Rivera, P. y Aránguiz, C. «El E-learning en el marco de la construcción de ciudadanía. Análisis contemporáneo a partir de la disociación entre modelos sociales y la crisis de sentido». Revista Chilena de Comunicación, volumen IV (1). Editorial Accesum. Santiago, Chile. 2011.

Rivera, P. «Impacto sociocultural de la formación profesional Elearning en las sociedades periféricas y desarrolladas.» Revista Chilena de Comunicación. Año II No2. 2010. Santiago de Chile. (2010):111-120.

Rivera, P. «La modernización en Chile: Compulsión o congestión social». Revista Perspectivas, Año XIII, № 19. Editorial LOM, número 19, Santiago de Chile, año 2008. (2008):37-56.

Sancho, J. M. (Coord.). La Tecnología: Un modo de transformar el mundo cargado de ambivalencia. En J. M. Sancho (Coord.): Para una Tecnología Educativa. Barcelona: Horsori. (1998):13-38.

Sangrà, A., Vlachopoulus, D, Cabrera, N., Bravo, S. Proyecto: Hacia una definición inclusiva del e-learning. Barcelona. ELearn Center. UOC. [Fecha de consulta: 30/ 01/2013]. Disponible en http://elconcept.uoc. edu/2011/12/ spanish-and-engish-versions-of-final.html, 2011.

Simonson, M., Salmadino, S., et al. Teaching and learning at a distance: Foundations of distance education ( $3^{\mathrm{a}} \mathrm{Ed}$.). Upper Saddle River, NJ: Merrill Prentice Hall. USA. 2006. 